\title{
Prevascularization with gelatin microspheres containing basic fibroblast growth factor enhances the benefits of cardiomyocyte transplantation
}

Yutaka Sakakibara, MD ${ }^{a}$

Kazunobu Nishimura, MD, PhD ${ }^{\mathrm{a}}$

Keiichi Tambara, $\mathrm{MD}^{\mathrm{a}}$

Masaya Yamamoto, $\mathrm{PhD}^{\mathrm{b}}$

Fanglin Lu, MD

Yasuhiko Tabata, $\mathrm{PhD}^{\mathrm{b}}$

Masashi Komeda, MD, PhD

From the Department of Cardiovascular Surgery, Graduate School of Medicine, ${ }^{\mathrm{a}}$ Kyoto University, and the Institute for Frontier Medical Sciences, ${ }^{\text {b }}$ Kyoto University, Kyoto, Japan.

This research was supported by a "Grantin-Aid" for Scientific Research (C) from the Ministry of Education, Culture, Sports, Science and Technology, and "Research for the Future" Program from the Japan Society for the Promotion of Science.

Received for publication Aug 27, 2001; revisions requested Oct 3, 2001; revisions received Oct 25, 2001; accepted for publication Oct 26, 2001.

Address for reprints: Masashi Komeda, MD, PhD, Professor, Graduate School of Medicine, Department of Cardiovascular Surgery, Kyoto University, 54 Kawaharacho Shogoin Sakyo-ku, Kyoto, Japan, 6068507 (E-mail: masakom@kuhp.kyotou.ac.jp).

J Thorac Cardiovasc Surg 2002;124:50-6

Copyright $(9) 2002$ by The American Association for Thoracic Surgery

0022-5223/2002 \$35.00+0 $\quad \mathbf{1 2 / 1 / 1 2 1 2 9 3}$

doi: $10.1067 / \mathrm{mtc} .2002 .121293$

Objective: The effects of cell transplantation on the ischemic failing heart have already been documented. However, the area in and around infarct regions is not a good environment for cells to survive in because they are exposed to poor conditions in which certain requirements cannot be adequately supplied. We therefore designed a study to investigate the efficacy of prevascularization in ischemic regions before cell transplantation.

Methods: Rats with myocardial infarction were randomized into 4 groups: 11 rats received a culture medium injection to the left ventricular wall (control group), 11 received fetal cardiomyocyte transplantation (TX group), 11 received gelatin hydrogel microspheres incorporating basic fibroblast growth factor (FGF group), and 11 received basic fibroblast growth factor pretreatment sequentially, followed by cardiomyocyte transplantation (FGF-TX group). Four weeks later, left ventricular function was assessed by means of echocardiography and cardiac catheterization.

Results: In the FGF and FGF-TX groups neovascularization was found in the scar tissue 1 week later. The TX, FGF, and FGF-TX groups showed better fractional shortening than the control group (TX, FGF, FGF-TX, and control: $28 \% \pm 4.4 \%$, $24 \% \pm 8.6 \%, 27 \% \pm 7.3 \%$, and $17 \% \pm 4.6 \%$, respectively; $P<.01$ ). Left ventricular maximum time-varying elastance was higher in the FGF-TX group than in the TX and FGF groups (FGF-TX, TX, and FGF: $0.52 \pm 0.23,0.30 \pm 0.08$, and $0.27 \pm 0.20 \mathrm{~mm} \mathrm{Hg} / \mu \mathrm{L}$, respectively; $P<.01$ ). Histologically, more transplanted cells survived in the FGF-TX group than in the TX group.

Conclusions: Prevascularization with basic fibroblast growth factor-incorporated microspheres enhances the benefits of cardiomyocyte transplantation. We expect that this system will contribute to regeneration medicine through its extensive application to other growth factors.

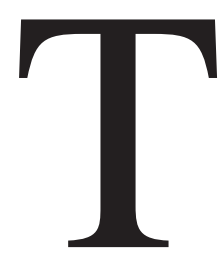

he number of patients with end-stage heart failure has been increasing. Presently, cardiac transplantation is believed to be the best treatment for most of these patients. However, the paucity of donors represents a significant problem. In North America, for instance, only less than $20 \%$ of transplantation candidates can receive donor are being sought. hearts. Therefore, alternative treatments for end-stage heart failure

In this context a number of studies have been reported on cell transplantation. $\mathrm{Li}$ and colleagues ${ }^{1,2}$ and Scorcin and coworkers ${ }^{3,4}$ showed that cardiomyocyte trans- 
plantation was effective in improving left ventricular (LV) function in rat myocardial infarction models. Other studies also reported the improvement of cardiac performance after transplantation of noncardiomyocyte cells (eg, skeletal myoblasts, smooth muscle cells, and mesenchymal stem cells derived from bone marrow)..$^{5-9}$ Although these findings implied the efficacy of cell transplantation, no studies, to the best of our knowledge, have focused on the adjuncts to improve the environment of transplanted cells exposed to ischemia, inflammation, and oxidative stress when they are transplanted into the ischemic or peri-ischemic regions.

Cell-growth factors, such as basic fibroblast growth factor (bFGF) and vascular endothelial growth factor (VEGF), are known to greatly contribute to neovascularization in the ischemic tissue area. They should be released over a long period of time at the site of action to obtain rich neovascularization. However, this has not always been successful, mainly because of their very short half-life in vivo. A number of strategies have been reported to overcome this problem by using protein release systems on the basis of polymer matrices. Because proteins are easily denatured and lose their biologic activities when exposed to harsh environments during the formulation process with a polymer matrix, it is necessary to exploit new formulation methods to induce slow release of proteins under mild conditions to minimize their denaturation. We previously documented that polymer hydrogels are a preferable matrix candidate for release of growth factors because of their biosafety and high inertness toward protein drugs. ${ }^{10}$ Subsequently, biodegradable gelatin microspheres incorporating bFGF have been developed with acidic gelatin hydrogels, enabling the release of bFGF at the site of action over a period of time adequate for effective neovascularization..$^{11,12}$

In the present study we investigated whether prevascularization of infarct or peri-infarct LV regions with this controlled release system of bFGF enhances the effects of cell transplantation in a rat myocardial infarction model.

\section{Materials and Methods}

Syngeneic Lewis rats weighing 250 to $290 \mathrm{~g}$ were used in this study. The study protocol was approved by the Kyoto University Ethics Committee for Animal Research. All animals received humane care in compliance with the "Principles of Laboratory Animal Care" formulated by the National Society for Medical Research and the "Guide for the Care and Use of Laboratory Animals" prepared by the Institute of Laboratory Animal Resources, National Research Council, and published by the National Academy Press.

\section{Preparation of bFGF-incorporating Gelatin Hydrogels} Human recombinant bFGF with an isoelectric point of 9.6 was supplied by Kaken Pharmaceutical Co (Tokyo, Japan). A gelatin sample with an isoelectric point of 5.0 was isolated from the bovine bone through the alkaline process (Nitta Gelatin Co, Osaka, Japan). Radioisotope $\mathrm{Na}^{125} \mathrm{I}(740 \mathrm{MBq} / \mathrm{mL}$ in $0.1 \mathrm{~N} \mathrm{NaOH}$ aque- ous solution) and N-succinimidyl-3-(4-hydroxy-3,5-di[ $\left.{ }^{125} \mathrm{I}\right]$-iodophenyl) propionate (Bolton-Hunter reagent, $147 \mathrm{MBq} / \mathrm{mL}$ ) were purchased from NEN Research Products (DuPont, Wilmington, Del). The bFGF microspheres were prepared as described previously. ${ }^{11}$ Briefly, gelatin microspheres were prepared through glutaraldehyde cross-linking of gelatin in aqueous solution dispersed in an oil phase. Then the microspheres were washed with acetone $\left(4^{\circ} \mathrm{C}\right)$ and recovered by means of centrifugation at $5000 \mathrm{rpm}$ and $4^{\circ} \mathrm{C}$ for 5 minutes. The obtained microspheres were finally washed by means of centrifugation with double-distilled water and freeze-dried. The average diameter of the microspheres was $10 \mu \mathrm{m}$. bFGF was radioiodinated according to the method of Greenwood and colleagues. ${ }^{13}$

\section{In Vivo Evaluation of bFGF Release and Gelatin Hydrogel Degradation}

Gelatin hydrogels incorporating ${ }^{125}$ I-labeled bFGF were implanted subcutaneously into the backs of mice. As a control, $100 \mu \mathrm{L}$ of aqueous solution of ${ }^{125}$ I-labeled bFGF was subcutaneously injected into the mouse back. The mice were killed at intervals, and the skin around the bFGF-implanted or injected site was cut into strips. The facia was thoroughly wiped off with filter paper. The remaining radioactivity of gelatin hydrogel, excised skin, and filter paper was measured on a gamma counter (ARC-301B; Aloka Co, Ltd, Tokyo, Japan) to evaluate the time profile of in vivo degradation of the gelatin hydrogels.

\section{Cardiomyocyte Isolation and Culture Procedures}

Ventricular cardiomyocytes were isolated from syngeneic Lewis fetal rat (Japan SLC Inc, Shizuoka, Japan) hearts and cultured as previously described. ${ }^{14}$ In brief, 20-day-old embryos hearts were removed and washed in phosphate-buffered saline solution. The minced ventricles were incubated in phosphate-buffered saline solution containing trypsin $(0.25 \%)$, collagenase $(0.05 \%)$, and glucose $(0.02 \%)$ at $37^{\circ} \mathrm{C}$ for 15 minutes. The cell suspension was transferred into Iscove's modified Dulbecco's medium (Gibco Laboratory, Life Technologies, Grand Island, NY) supplemented with $10 \%$ fetal bovine serum, $0.1 \mathrm{mmol} / \mathrm{L} \beta$-mercaptoethanol, 100 $\mathrm{U} / \mathrm{mL}$ penicillin, and $100 \mu \mathrm{g} / \mathrm{mL}$ streptomycin. The cell suspension was centrifuged at $500 \mathrm{~g}$ for 5 minutes, and the cell pellet was resuspended in culture medium. At this stage, cardiomyocytes were purified by using a preplating method. Before each transplantation, all cells were labeled with fluorescent dyes with a PKH 26 Red Fluorescent Cell Linker Kit (Sigma Chemical Co, St. Louis, Mo) for general cell membrane labeling.

\section{Chronic Myocardial Infarction Model}

Male Lewis rats were orally intubated into the trachea after being anesthetized with ethyl ether gas. Anesthesia was maintained during the operation with $1 \%$ to $1.5 \%$ isoflurane. The proximal left anterior descending coronary artery was ligated proximally with a 5-0 polypropylene suture. ${ }^{15,16}$ An ST-segment elevation on electrocardiography and color changes in the LV muscle were noted in all rats. Four weeks after the LAD ligation, infarction size and cardiac function were evaluated by means of echocardiography, as described below. ${ }^{17,18}$

\section{Experimental Groups}

Four weeks after the LAD ligation, the hearts were exposed by a lateral rethoracotomy after achievement of general anesthesia, as 
$<$ TX, FGF, Control group >

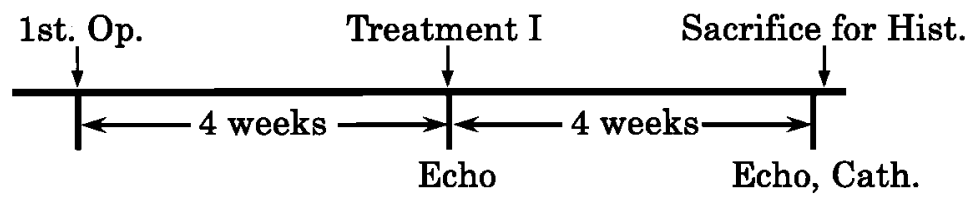

$<$ FGF-TX group >

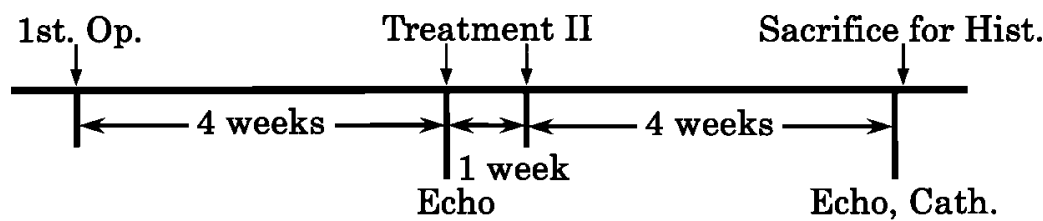

Figure 1. Experimental protocol. $1 s t ~ 0 p$, Ligation of the left anterior descending coronary artery; Treatment I, culture medium injection or fetal cardiomyocyte transplantation or injection of gelatin hydrogel microspheres incorporating bFGF (bFGF microsphere injection); Treatment II, bFGF microsphere injection followed by fetal cardiomyocyte transplantation; Echo, echocardiography; Cath, measurement of the LV pressure with a micromanometertipped catheter; and Hist, histologic study.

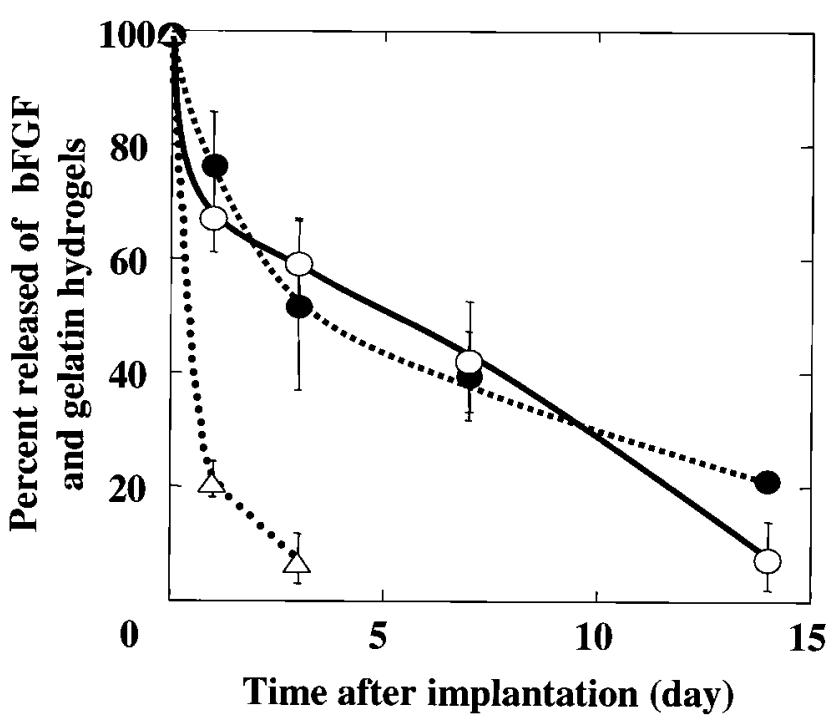

Figure 2. In vivo release of bFGF and gelatin hydrogels. Patterns of declining radioactivity in the back subcutis of mice after subcutaneous implantation of ${ }^{125}$ I-labeled bFGF in solution (open triangles), in hydrogels (open circles), and in ${ }^{125}$ I-labeled gelatin hydrogels (filled circles).

described in the myocardial infarction model above. Forty-four rats were randomized into 4 groups: 11 rats received an intramyocardial injection of only culture medium (control group), 11 received fetal cardiomyocyte transplantation (TX group), 11 received an injection of gelatin hydrogel microspheres incorporating bFGF (bFGF microspheres, FGF group), and 11 received bFGF microsphere injection, followed by sequential fetal cardiomyocyte transplantation 1 week later (FGF-TX group).

Cell Transplantation or bFGF Microsphere Injection In each group the volume of the injected solution was $50 \mu \mathrm{L}$. In the TX and FGF groups $6 \times 10^{6}$ cultured fetal cardiomyocytes and bFGF microspheres, respectively, were injected with a fine syringe into the center of the scar tissue of the LV free wall. In the FGF-TX group bFGF microsphere injection and the following cardiomyocyte transplantation were performed by using the same method as above.

\section{Assessment of the LV Function}

LV function was assessed by means of echocardiography (SONOS 5500 Imaging System; Agilent Technologies, Andover, Mass). Images were recorded from the left parasternal windows in the right lateral decubitus position. The following parameters were measured and derived from the B- and M-mode tracing: LV end-diastolic dimension (in millimeters), LV end-systolic dimension (in millimeters), fractional shortening (percentage), and fractional area change. ${ }^{19}$ Infarction size was estimated by using the percentage of the akinetic region divided by the LV endocardial circumference on a midventricular short-axis view at end diastole. ${ }^{20}$ One day after echocardiographic evaluation, all rats had more precise assessment of global LV function before death. ${ }^{17,18} \mathrm{~A} 2 \mathrm{Fr}$ micromanometer-tipped catheter (Millar Instruments Inc, Houston, Tex) was inserted into the LV through the right carotid artery, and a $3 \mathrm{~F}$ occlusion balloon catheter was inserted into the inferior vena cava (IVC) through the right iliac vein to occlude the IVC. LV pressure and its first time derivative $(\mathrm{dP} / \mathrm{dt})$ were continuously monitored through the arterial catheter by using a multiple recording system. Two-dimensionally targeted M-mode echocardiograms were obtained along the short-axis view of the left ventricle at the level of the papillary muscles to calculate LV systolic 


\section{LVEmax}



LVEDP

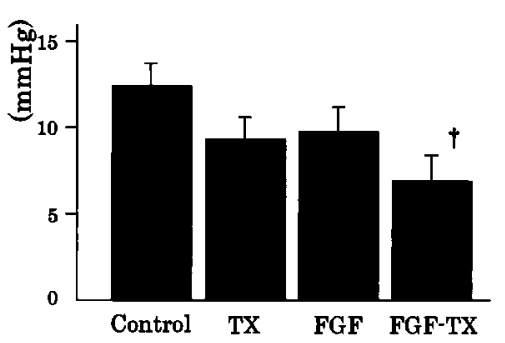

Tau

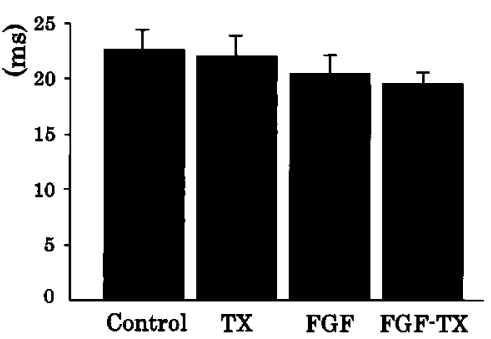

Figure 3. Indices of LV maximum time-varying elastance (LVEmax), LV end-diastolic pressure (LVEDP), and $\tau$ in hearts of mice from the control, TX, FGF, and FGF-TX groups 4 weeks after each treatment. Error bars show SEM. The FGF-TX group shows a higher LVEmax than the other groups $\left(^{*} \boldsymbol{P}<.05\right.$ and ${ }^{*} \boldsymbol{P}<.01$ vs FGF-TX group). The FGF-TX group shows a lower LVEDP than the other groups ( $P<.05$ vs control group). There was no significant difference in $\tau$ among the 4 groups.

TABLE I. Cardiac function data obtained by means of echocardiography among the control, TX, FGF, and FGF-TX groups

\begin{tabular}{|c|c|c|c|c|}
\hline & Control group & TX group & FGF group & FGF-TX group \\
\hline \multicolumn{5}{|l|}{ Before treatment } \\
\hline LVDd (mm) & $10.3 \pm 0.8$ & $10.1 \pm 0.7$ & $9.7 \pm 0.9$ & $9.7 \pm 0.9$ \\
\hline LVDs (mm) & $7.9 \pm 2.2$ & $7.8 \pm 0.9$ & $7.9 \pm 1.1$ & $7.8 \pm 1.0$ \\
\hline FS $(\%)$ & $18.8 \pm 5.4$ & $22.4 \pm 5.4$ & $19.1 \pm 6.7$ & $19.8 \pm 4.1$ \\
\hline FAC $(\%)$ & $25.9 \pm 3.3$ & $26.8 \pm 6.6$ & $28.2 \pm 6.5$ & $28.2 \pm 4.6$ \\
\hline HR (beats/min) & $373 \pm 32$ & $364 \pm 31$ & $359 \pm 47$ & $372 \pm 43$ \\
\hline Infarction size (\%) & $27.4 \pm 2.9$ & $27.0 \pm 4.1$ & $30.8 \pm 5.3$ & $29.3 \pm 4.0$ \\
\hline \multicolumn{5}{|l|}{ After treatment } \\
\hline LVDd (mm) & $10.7 \pm 0.6$ & $10.0 \pm 1.1$ & $9.5 \pm 0.7 \dagger$ & $9.6 \pm 0.6^{*}$ \\
\hline LVDs (mm) & $8.8 \pm 0.7$ & $7.2 \pm 0.9 \dagger$ & $7.2 \pm 1.1 \dagger$ & $7.0 \pm 1.0 \dagger$ \\
\hline FS $(\%)$ & $17.3 \pm 4.6$ & $28.4 \pm 4.4 \dagger$ & $24.5 \pm 8.6$ & $27.4 \pm 7.3 \dagger$ \\
\hline FAC $(\%)$ & $22.5 \pm 4.3$ & $34.0 \pm 11.9 \dagger$ & $32.6 \pm 6.7^{*}$ & $41.7 \pm 6.8 \dagger \ddagger$ \\
\hline HR (beats/min) & $357 \pm 31$ & $374 \pm 36$ & $363 \pm 40$ & $376 \pm 56$ \\
\hline Infarction size (\%) & $27.5 \pm 3.5$ & $27.0 \pm 4.6$ & $28.1 \pm 5.2$ & $24.9 \pm 5.0$ \\
\hline
\end{tabular}

All values are shown as the means \pm SD.

$L V D$ d, Left ventricular end-diastolic dimension; $L V D s$, end-systolic dimension; $F S$, fractional shortening; $F A C$, fractional area change; $H R$, heart rate; Infarction size, the percentage of akinetic endocardial length to the whole left ventricular endocardial circumference.

${ }^{*} P<.05$ vs control group.

$\dagger P<.01$ vs control group.

$\ddagger P<.05$ vs FGF group.

volume from LV end-systolic dimension by the cube formula. During IVC occlusion with the balloon, LV pressure waveforms and M-mode tracings were simultaneously recorded on the same monitor. The end-systolic pressure-volume points obtained from echocardiography and cardiac catheterization were subjected to least-squared linear regression, and the LV maximum time-varying elastance (in millimeters of mercury per microliter) was calculated as the slope of the fitted line. The time constant of isovolumic relaxation $(\tau$ [in milliseconds] $=\mathrm{LV}$ pressure at peak negative/ $-\mathrm{dp} / \mathrm{dt}$ ) as an index of global systolic and diastolic function was calculated. LV end-diastolic pressure (in millimeters of mercury) was measured at the same time. Figure 1 shows the study protocol, including the operation and sampling points in each group.

\section{Histologic Study}

After final assessment, all rats were killed, and the hearts were removed for histologic study. The specimens were separated in halves by the short-axis section, including the injection point; one part was fixed with $10 \%$ formalin for hematoxylin and eosin staining, and the other part was cryopreserved for staining with PKH26. Then sections were microscopically examined with the use of fluorescence microscopy for PKH26 dye. Five rats with bFGF microsphere injection and 5 with culture medium injection only were also prepared as above in addition to the follow-up study groups to quantify the neovascularization associated with bFGF pretreatment. They were killed, and the hearts were extirpated a week after each treatment. Heart sections of $10-\mu \mathrm{m}$ thickness were prepared and 

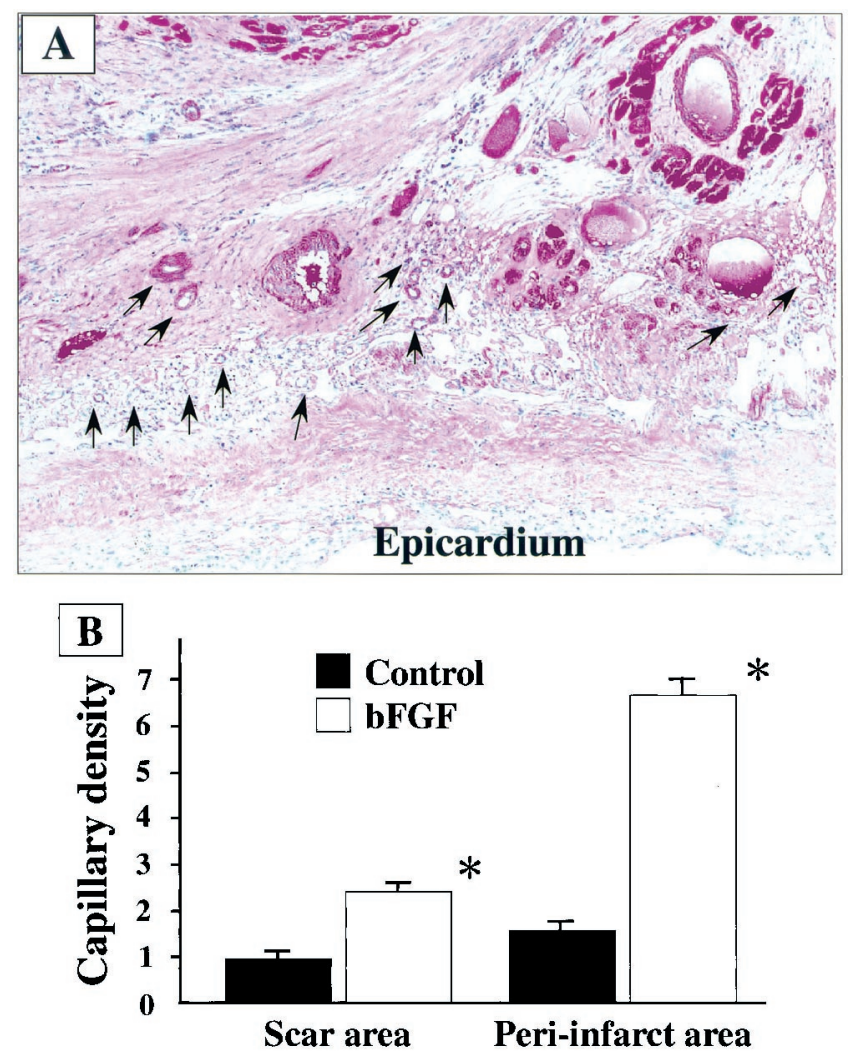

Figure 4. A, Neovascularization in the scar tissue of the left ventricle free wall 1 week after the injection of bFGF microspheres. Arrow indicates neovessels. (Hematoxylin and eosin, original magnification $100 \times$.) B, Capillary density in scar and peri-infarct area in each treatment group $(n=5)$ is expressed as the number of capillary vessels per high-power field. Data are shown as means \pm SEM. The bFGF treatment groups have a higher capillary vessel density than the control group in both areas $\left({ }^{*} P<.001\right)$.

stained with hematoxylin and eosin. The vessels per unit of area (200 $\mu \mathrm{m}^{2}$ ) in the scar and the peri-infarct region were counted in 5 fields per each slide by 2 blinded pathologists. The average number of the vessels in one field was used for statistical analysis.

\section{Data Analysis}

All values were shown as the mean values \pm SD. The pairwise comparisons of individual group means were conducted by means of the Tukey test. Statistical analyses were performed with Statview for Windows version 5.0 (SAS Institute Inc, Cary, NC).

\section{Results}

Coronary artery ligation was performed in a total of 70 rats, of which 12 died within a week. Four weeks later, echocardiography was performed on the survivors. Among them, 4 had no or a small myocardial infarction (infarct size, $<20 \%$ ), and 10 had large infarctions (infarct size, $>40 \%$ ). The rats with moderate-size myocardial infarctions in the anterior LV wall $(\mathrm{n}=44)$ were then enrolled in this study. Each rat in the FGF and FGF-TX groups died immediately after the injection of bFGF microspheres, and data from these animals were excluded from analysis.

\section{In Vivo Release of bFGF}

Figure 2 shows the pattern of decreasing residual radioactivity after subcutaneous implantation in the backs of mice in gelatin hydrogels incorporating different ${ }^{125}$ I-labeled bFGF. More than $80 \%$ of the bFGF injected as solution was cleared from the injected site within 1 day. In marked contrast there was prolonged release of bFGF from the hydrogel at the site of implantation. In addition, there was a strong correlation between the patterns of in vivo bFGF release and hydrogel degradation, as seen in Figure 2. This result suggests that bFGF, together with a fragment of gelatin molecules, is released as a result of hydrogel degradation.

\section{Characterization and Identification of Graft Cells}

Most of the isolated cells derived from fetal rat hearts were observed to beat in culture medium and were composed of more than $90 \%$ of cardiomyocytes, as determined by means of immunostaining with alpha-sarcomeric muscle actin. Transplanted cells were detected with PKH26 red fluorescent dye in each recipient heart.

\section{In Vivo Cardiac Function}

There was no significant difference among the 4 groups in cardiac function and infarction size before treatment. Four weeks after each treatment, LV end-diastolic dimension in the FGF and FGF-TX groups was smaller than in the control group. The TX, FGF, and FGF-TX groups had significantly smaller LV end-systolic dimension than the control group. These 3 groups had better fractional shortening and fractional area change than the control group. These findings are summarized in Table I. LV maximum time-varying elastance in the FGF-TX group was $430 \%(P<.01)$ higher than in the control group and $172 \%(P<.05)$ higher than in the TX group (Figure 3). LV end-diastolic pressure in the FGF-TX group was the lowest and 55\% lower than that in the FGF-TX group (Figure 3). There was no significant difference in $\tau$ among the 4 groups (Figure 3 ).

\section{Histology in the Recipient Heart}

Microscopic examination showed the findings below. There were many neovessels in and around the scar tissue 1 week after the injection of bFGF microspheres (Figure 4). Vascular densities in the scar and peri-infarction regions in the specimens from the rats injected with bFGF microspheres were higher than those from rats without bFGF treatment $(P<.001$, Figure 4$)$. Four weeks after cell transplantation, transplanted cardiomyocytes labeled with PKH26 red fluorescent dye were detected in the scar area. Whereas most of the transplanted cells were found in the peri-infarction re- 

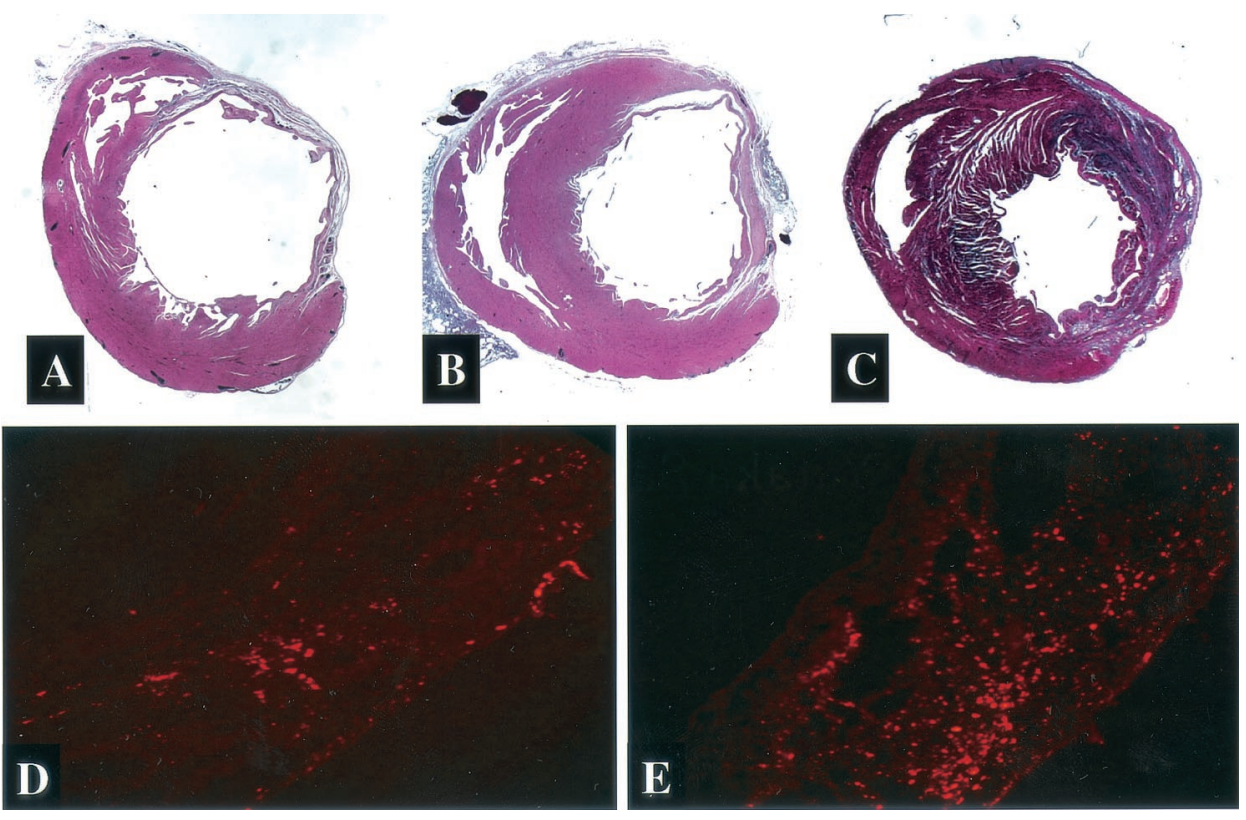

Figure 5. A-C, Histologic findings 4 weeks after each treatment: A, control group; B, TX group; C, FGF-TX group. (Hematoxylin and eosin staining, original magnification $1 \times$.) D and $E$, Fluorescent image of transplanted cells labeled with PKH26 red fluorescent dye (D, peri-infarct area in the TX group; $E$, middle of the myocardial infarction in the FGF-TX group). (Original magnification $40 \times$.)

gions in the TX group, transplanted cells in the FGF-TX group were detected in all scar areas, including infarct regions, even in the middle of the scar (Figure 5, $D$ and $E$ ). Many vessels were found in and around transplanted tissue in the FGF-TX group. The infarct LV wall was thicker in the TX, FGF, and FGF-TX group than in the control group (Figure 5, A-C).

\section{Discussion}

In this study cardiomyocyte transplantation was effective for the ischemic failing heart. However, transplanted cells in the control group survived only in the peri-infarct regions and not in the scar area. On the other hand, in the FGF-TX group many cardiomyocytes were found in the scar tissue, and cardiac function in that group was much improved compared with that in the TX group. We speculate that the following mechanisms may account for these findings. First, bFGF may have enhanced the viability of transplanted cardiomyocytes because many more transplanted cells were observed in histologic sections. It may be that in the FGF-TX group neovascularization induced around the site of infarction provided adequate oxygen, nutrition, and other substances, such as growth factors. Second, bFGF may have exerted several kinds of direct effects on transplanted fetal cardiomyocytes. Pasumarthi and colleagues ${ }^{21}$ reported that bFGF may stimulate DNA synthesis and myocyte proliferation and differentiation during development, favoring a more fetal program of contractile gene expression. ${ }^{22,23}$
The results of the present study suggested the importance of securing an adequate blood supply in the infarct and peri-infarct LV regions for the transplanted cells to survive. Recently, it was reported that myocardial angiogenesis by means of gene transfer with transplanted cells improved cardiac function in ischemic failing hearts. However, with the genetic method, the effect of angiogenesis cannot be started immediately after cell transplantation. In addition, gene therapy may not be safe because of its side effects. In view of these considerations, prevascularization with the controlled release system with bFGF microspheres may provide a unique environment that is favorable for various transplanted cells in the treatment of heart failure with myocardial infarction.

There are several limitations to the clinical application of the present methodology. First, it may be technically demanding to inject bFGF microspheres into the scarred LV wall, which is very thin. In our experiments 2 rats died during the operative procedure. These deaths were probably caused by misinjection of bFGF microspheres, with subsequent systemic embolization. Gelatin particles are 10 to 16 $\mu \mathrm{m}$ in diameter, and in FGF solution they combined with bFGF to form large particles. Because of this property, we should be very careful to avoid the intraluminal administration of the microspheres. The risk of misinjection should be reduced when microspheres are injected into the myocardium of human subjects or large animals. Second, considering combined therapy, it may not be feasible to open the 
chest twice to inject bFGF and, later, myocardial cells. Although in the clinical setting it might seem more practical to inject bFGF and transplanted cells simultaneously, theoretically there should be some time lag to allow for the development of neovascularization. Hence further research is necessary to investigate new methods to inject bFGF microspheres without operation.

Various methods have been reported for the stimulation of angiogenesis in infarcted regions. These include the introduction of genes encoding VEGF or bFGF and transplantation of interstitial cells from bone marrow and vascular endothelial cells. ${ }^{24,25}$ We recently showed that gelatin hydrogel microspheres incorporating bFGF facilitate angiogenesis in infarcted regions. ${ }^{11,12}$ Although bFGF has a potent angiogenic function, its half-life in vivo is very short. In addition, it is likely that the injected free bFGF rapidly flows away from the injection site. Therefore the injection of bFGF in solution is not very effective in inducing vascularization. ${ }^{11,12,26}$ In the method with bFGF-incorporating gelatin hydrogel microspheres, however, slowly released bFGF exerts its activities and stimulates angiogenesis locally, with the gelatin hydrogel microspheres being absorbed into the living tissue. This system may induce angiogenesis sufficient to supply essential requirements for cell survival before cell transplantation. With this system, biologically active growth factors other than bFGF, such as hepatocyte growth factor and VEGF, can be released as a result of in vivo degradation of the hydrogels. Because the release profile can be controllable by changing the water content of gelatin hydrogels, ${ }^{10,11}$ we believe that this controlled release system can contribute greatly to the progress of regeneration medicine through its extensive application to other growth factors.

Consultation concerning data analysis was provided by Dr Chikuma Hamada (Department of Pharmacoepidemiology, School of Public Health, Kyoto University, Japan). We also thank Dr Ren-Ke Li and Dr Richard D Weisel (the Division of Cardiac Surgery at the University of Toronto General Hospital, Canada) for their assistance.

\section{References}

1. Li RK, Jia ZQ, Weisel RD, Mickle DA, Zhang J, Mohabeer MK, et al. Cardiomyocyte transplantation improves heart function. Ann Thorac Surg. 1996;62:654-60.

2. Li RK, Weisel RD, Mickle DA, Jia ZQ, Kim EJ, Sakai T, et al. Autologous porcine heart cell transplantation improved heart function after a myocardial infarction. J Thorac Cardiovasc Surg. 2000;119: $62-8$.

3. Scorsin M, Hagege AA, Marotte F, Mirochnik N, Copin H, Barnoux $\mathrm{M}$, et al. Does transplantation of cardiomyocytes improve function of infarcted myocardium? Circulation. 1997;96(Suppl):II-188-93.

4. Scorsin M, Hagège A, Vilquin JT, Fiszman M, Marotte F, Samuel JL, et al. Comparison of the effects of fetal cardiomyocyte and skeletal myoblast transplantation on postinfarction left ventricular function. J Thorac Cardiovasc Surg. 2000;119:1169-75.
5. Tomita S, Li RK, Weisel RD, Mickle DA, Kim EJ, Sakai T, et al. Autologous transplantation of bone marrow cells improves damaged heart function. Circulation. 1999;100(Suppl):II-247-56.

6. Li RK, Jia ZQ, Weisel RD, Merante F, Mickle DA. Smooth muscle cell transplantation into myocardial scar tissue improves heart function. J Mol Cell Cardiol. 1999;31:513-22.

7. Sakai T, Li RK, Weisel RD, Mickle DA, Jia ZQ, Tomita S, et al. Fetal cell transplantation: a comparison of three cell types. $J$ Thorac Cardiovasc Surg. 1999;118:715-25.

8. Taylor DA, Atkins BZ, Hungspreugs P, Jones TR, Reedy MC, Hutcheson KA, et al. Regenerating functional myocardium: improved performance after skeletal myoblast transplantation. Nat Med. 1998; 4:929-33.

9. Suzuki K, Brand NJ, Smolenski RT, Jayakumar J, Murtuza B, Yacoub $\mathrm{MH}$. Development of a novel method for cell transplantation through the coronary artery. Circulation. 2000;102(Suppl):II-359-64.

10. Tabata Y, Masaya Y, Ikada Y. Biodegradable hydrogels for bone regeneration through growth factors release. Pure \& Appl Chem. 1998;70:1277-82.

11. Tabata Y, Ikada Y. Protein release from gelatin matrices. Adv Drug Deliv Rev. 1998;31:287-301.

12. Tabata Y, Hijikata S, Muniruzzaman MD, Ikada Y. Neovascularization effect of biodegradable gelatin microspheres incorporating basic fibroblast growth factor. J Biomater Sci Polym Ed. 1999;10:79-94.

13. Greenwood FC, Hunter WM, Gglover TC. The preparation of ${ }^{131} \mathrm{I}-$ labeled human growth hormone of high specific radioactivity. Biochem J. 1963;89:114-23.

14. Li RK, Mickle DA, Weisel RD, Zhang J, Mohabeer MK. In vivo survival and function of transplanted rat cardiomyocytes. Circ Res. 1996;78:283-8.

15. Isgaard J, Kujacic V, Jennische E, Holmang A, Sun XY, Hedner T, et al. Growth hormone improves cardiac function in rats with experimental myocardial infarction. Eur J Clin Invest. 1997;27:517-25.

16. Pfeffer MA, Pfeffer JM, Steinberg C, Finn P. Survival after an experimental myocardial infarction: beneficial effects of long-term therapy with captopril. Circulation. 1985;72:406-12.

17. Nishina T, Nishimura K, Yuasa S, Miwa S, Nomoto T, Sakakibara Y, et al. Initial effects of the left ventricular repair surgery may not last long in a rat ischemic cardiomyopathy model. Circulation. 2001; 104(Suppl):I-241-5.

18. Nishina T, Nishimura K, Yuasa S, Miwa S, Sakakibara Y, Ikeda T, et al. A rat model of ischemic cardiomyopathy for investigating left ventricular volume reduction surgery. J Card Surg. In press.

19. Burrell LM, Chan R, Phillips PA, Calafiore P, Tonkin AM, Johnston CI. Validation of an echocardiographic assessment of cardiac function following moderate size myocardial infarction in the rat. Clin Exp Pharmacol Physiol. 1996;23:570-2.

20. Sahn DJ, DeMaria A, Kisslo J, Weyman A. Recommendations regarding quantitation in M-mode echocardiography: results of a survey of echocardiographic measurements. Circulation. 1978;58:1072-83.

21. Pasumarthi SKB, Doble BW, Kardami E. Overexpression of CUG-and AUG-initiated forms of basic fibroblast growth factor in cardiac myocytes results in similar effects on mitosis and protein synthesis but distinct nuclear morphologies. J Mol Cell Cardiol. 1994;26:1045-60.

22. Parker TG, Schneider MD. Growth factors, proto-oncogenes, and plasticity of the cardiac phenotype. Annu Rev Physiol. 1991;53:179200.

23. Kardami E. Stimulation and inhibition of cardiac myocyte proliferation in vitro. Mol Cell Biochem. 1990;92:129-35.

24. Yau TM, Sarjeant JM, Fung K, Weisel RD, Mickele DA, Li RK. Enhanced myocardial angiogenesis by gene transfer using transplanted cells. Circulation. 2001;104(Suppl):I-218-22.

25. Kalka C, Masuda H, Takahashi T, Kalka-Moll WM, Silver M, Kearney M, et al. Transplantation of ex vivo expanded endothelial progenitor cells for therapeutic neovascularization. Proc Natl Acad Sci U S A. 2000;97:3422-7.

26. Tabata Y, Nagano A, Ikada Y. Biodegradation of hydrogel carrier incorporating fibroblast growth factor. Tissue Eng. 1999;5:127-38. 